\title{
Surfactant protein B and A concentrations are increased in neonatal pneumonia
}

\author{
Sara D'Aronco' ${ }^{1}$, Manuela Simonato ${ }^{1,2}$, Luca Vedovelli ${ }^{2}$, Aldo Baritussio ${ }^{3}$, Giovanna Verlato' ${ }^{1}$, Stefano Nobile ${ }^{4}$, Chiara Giorgetti ${ }^{4}$, \\ Matteo Nespeca ${ }^{4}$, Virgilio P. Carnielli ${ }^{4}$ and Paola E. Cogo ${ }^{5}$
}

\begin{abstract}
BACKGROUND: Term newborns with pneumonia show a reduced pulmonary compliance due to multiple and illdefined factors. Surfactant proteins'(SPs) changes could have a role in the reduced compliance but the matter is still unsettled. The aim of this study was to clarify the meaning of SPs changes during pneumonia in term newborns.
\end{abstract}

METHODS: In 28 term ventilated newborns, 13 with pneumonia and 15 with no lung disease, we measured SP-B, SP-A, disaturated-phosphatidylcholine (DSPC), and total phospholipids (PL) concentrations in tracheal aspirates at intubation and close to extubation. We also measured DSPC kinetics using (U- $\left.{ }^{13} \mathrm{C}-\mathrm{PA}\right)$ dipalmitoyl-phosphatidylcholine.

RESULTS: At baseline, SP-B, expressed as \% of PL, was significantly different between the groups, being 3.5 -fold higher in pneumonia than controls. Conversely, SP-A did not vary between the groups. At extubation, SP-B and SP-A concentrations had decreased significantly in newborns with pneumonia, while there was no significant change in controls. DSPC $t_{1 / 2}$ was significantly shorter in the pneumonia group (11.8 (5.5-19.8) h vs. $26.6(19.3-63.6) h, P=0.011)$.

CONCLUSION: In term newborns with pneumonia, SP-B increases with respect to $\mathrm{PL}$, and DSPC is turned over at a faster rate. Disease's resolution is associated with the restoration of the normal ratio between SP-B and PL.

A lveolar surfactant is essential for lung stability and its composition and functional state are altered during pneumonia and acute respiratory distress syndrome $(1,2)$. Pulmonary surfactant also plays an important role on the innate immune system by enhancing pathogen clearance and by regulating immune-cell functions (3).

Surfactant proteins (SPs) represent less than $10 \%$ of total surfactant weight but they play a pivotal role on its function. SP-B is a hydrophobic protein that is considered to be the most important protein for sustaining respiratory physiology (4). SP-B enhances lipid insertion into the monolayer at the air/liquid interface (5), it is involved in the formation of tubular myelin (6), and it may also be part of the host defense mechanisms (7). SP-A, a pulmonary collectin, plays an important role in lung innate immune system (8) but it is also involved in the regulation of surfactant homeostasis and in the formation of tubular myelin (7).

Respiratory failure in newborns with respiratory distress syndrome is due to surfactant deficiency (9), while the reduced pulmonary compliance observed in term newborns at the onset of pneumonia still does not have a clear explanation.

Understanding of SPs changes in children with lung infection is limited and sometimes discordant.

Reduced amounts of SP-A have been found in infants with acute viral bronchiolitis, including forms due to respiratory syncytial virus $(10,11)$ and bacterial and viral pneumonia (12). Kerr et al. (11) found decreased levels of SP-B in the bronchoalveolar lavage fluid (BALF) of children with severe respiratory syncytial virus bronchiolitis, while LeVine et al. (12) found concentrations that did not differ from controls in infants with bacterial and viral pneumonia. Moreover, a recent study in children with acute lung injury, showed no change in either BALF SP-B and SP-A concentrations (13).

In this paper, we aimed to measure SPs changes during pneumonia. We studied term newborns with and without pneumonia who required mechanical ventilation. We compared the concentrations of SP-B and SP-A in tracheal aspirates (TAs) obtained soon after intubation and after clinical improvement just before extubation, in both groups. We also investigated if SP-B and SP-A levels correlate with the degree of respiratory failure and with disaturated-phosphatidylcholine (DSPC) turnover rate, that is known to be increased, with shorter $t_{1 / 2}$ values, during various acute lung diseases $(14,15)$.

\section{RESULTS}

We studied 13 newborns with pneumonia and 15 controls with no lung disease. Eight controls were extubated immediately after surgery and therefore, for these patients, we collected only one TA sample.

SP-B, SP-A, DSPC, and total phospholipids (PL) concentrations in epithelial lining fluid (ELF) at the start of the study could be measured in 11 out of 13 newborns with pneumonia and in 14 out of 15 control newborns.

\footnotetext{
'Department of Women's and Children's Health, University of Padua, Padua, Italy; ${ }^{2}$ PCare Laboratory, Pediatric Research Institute "Città della Speranza", Padua, Italy; ${ }^{3}$ Department of Medicine, University of Padua, Padua, Italy; ${ }^{4}$ Division of Neonatology, Department of Clinical Sciences, Polytechnic University of Marche and Azienda

Ospedaliero-Universitaria Ospedali Riuniti, Ancona, Italy; ${ }^{5}$ Pediatric Cardiac Anesthesia/Intensive Care Unit, Department of Pediatric Cardiology and Cardiac Surgery,

Bambino Gesù Children's Hospital, Rome, Italy. Correspondence: Sara D’Aronco (lab1@pediatria.unipd.it)

Received 27 October 2014; accepted 2 April 2015; advance online publication 22 July 2015. doi:10.1038/pr.2015.123
} 
Close-to-extubation, and at least after $6 \mathrm{~h}$ from the previous TA, SP-B, SP-A, DSPC, and PL could be measured in 11 out of 13 newborns with pneumonia and in 7 out of 15 controls. At this timepoint, we could not express the amount of SPs as weight/volume of ELF because the arterial line had already been removed.

DSPC kinetic was measured in 19 newborns from whom additional parental consent was obtained, 12 of the pneumonia group and 7 controls. In three newborns (two with pnemonia and one with no lung disease) only DSPC kinetics was measured because the sample was too scarce to perform SPs' analysis.

Clinical characteristics and respiratory parameters of the study patients, are reported in Table 1 . None of the study patients received exogenous surfactant.

Table 1. Clinical characteristics and respiratory parameters in pneumonia and control group

\begin{tabular}{|c|c|c|c|}
\hline & Pneumonia group & Control group & $P$ \\
\hline Gestational age ${ }^{\mathrm{a}}$ (weeks) & $38.9 \pm 1.4$ & $38.6 \pm 1.4$ & 0.586 \\
\hline Birth weight $^{\mathrm{a}}(\mathrm{g})$ & $3226 \pm 605$ & $3064 \pm 650$ & 0.504 \\
\hline Age at study start ${ }^{\mathrm{b}}$ (days) & $2.3(1.0-5.4)$ & $2.4(1.0-8.0)$ & 0.730 \\
\hline $\begin{array}{l}\text { C-reactive protein at } \\
\text { study start }{ }^{\mathrm{b}}(\mathrm{mg} / \mathrm{l})\end{array}$ & $26.1(10.4-92.9)$ & $3.3(2.9-4.9)$ & 0.003 \\
\hline $\begin{array}{l}\text { Mechanical ventilation at } \\
\text { study start }{ }^{\mathrm{b}}(\mathrm{h})\end{array}$ & $44(12$ & $24(18-52)$ & 0.593 \\
\hline $\mathrm{FiO}_{2}$ at study start ${ }^{\mathrm{b}}$ & $0.50(0.25-0.60)$ & $0.21(0.21-0.23)$ & 0.001 \\
\hline $\mathrm{PaO}_{2}$ at study start ${ }^{\mathrm{a}}$ & $67.8 \pm 15.4$ & $79.7 \pm 17.7$ & 0.060 \\
\hline Ol at study start ${ }^{\mathrm{b}}$ & $5.7(3.1-7.5)$ & $1.4(1.2-1.7)$ & $<0.001$ \\
\hline $\mathrm{AaDO}_{2}$ at study start ${ }^{\mathrm{b}}$ & $194.5(75.6-307.2)$ & $20.1(16.6-36.6)$ & $<0.001$ \\
\hline $\mathrm{PaO}_{2} / \mathrm{FiO}_{2}$ at study start ${ }^{\mathrm{a}}$ & $173.9 \pm 84.3$ & $361.7 \pm 110.6$ & $<0.001$ \\
\hline PIP at study start ${ }^{\mathrm{a}}\left(\mathrm{cm} \mathrm{H}_{2} \mathrm{O}\right)$ & $20.8 \pm 3.6$ & $15.8 \pm 2.4$ & 0.001 \\
\hline PEEP at study start $\left(\mathrm{cm} \mathrm{H}_{2} \mathrm{O}\right)$ & $4.0 \pm 0.6$ & $3.2 \pm 0.9$ & 0.035 \\
\hline MAP at study start ${ }^{\mathrm{a}}\left(\mathrm{cm} \mathrm{H}_{2} \mathrm{O}\right)$ & $8.7 \pm 3.1$ & $5.2 \pm 1.2$ & $<0.001$ \\
\hline
\end{tabular}

$\mathrm{AaDO}_{2}$, alveolar-arterial oxygen gradient; $\mathrm{FiO}_{2}$, fraction of inspired oxygen; MAP, mean airway pressure; $\mathrm{Ol}$, oxygenation index; $\mathrm{PaO}_{2}{ }^{2}$ arterial $\mathrm{pO}_{2}$; $\mathrm{PIP}$, peak inspiratory pressure; PEEP, positive end expiratory pressure.

${ }^{a}$ Data presented as mean \pm SD. ${ }^{b}$ Data presented as median (IQR).

a

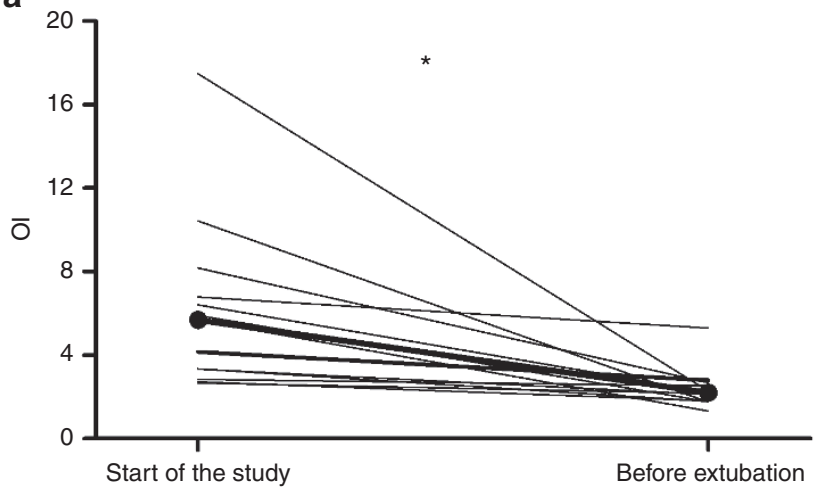

The two study groups were comparable for gestational age, birth weight, age, and hours of mechanical ventilation at the study start, while significant differences were observed in C-reactive protein, fraction of inspired oxygen $\left(\mathrm{FiO}_{2}\right)$, oxygenation index $(\mathrm{OI})$, alveolar-arterial oxygen gradient $\left(\mathrm{AaDO}_{2}\right)$, $\mathrm{PaO}_{2} / \mathrm{FiO}_{2}$ ratio, peak inspiratory pressure, positive end expiratory pressure, and mean airway pressure (MAP) at the start of the study. A tendency to lower values was observed for $\mathrm{PaO}_{2}$ in the pneumonia group compared to controls.

By paired analysis, $\mathrm{FiO}_{2}, \mathrm{OI}, \mathrm{AaDO}_{2}$, peak inspiratory pressure, and MAP decreased significantly during the study in the pneumonia group: from $0.50(0.25-0.60)$ to $0.25(0.22-0.29)$ $(P=0.008)$; from $5.7(3.1-7.5)$ to $2.2(1.8-2.7)(P=0.002)$, from 194.5 (75.6-307.2) to $45.6(37.9-77.1)(P=0.006)$, from $20.8 \pm 3.6$ to $17.2 \pm 2.2(P=0.005) \mathrm{cm} \mathrm{H}_{2} \mathrm{O}$, from $8.7 \pm 3.1$ to $6.1 \pm 0.7(P=0.003) \mathrm{cm} \mathrm{H}_{2} \mathrm{O}$ respectively. $\mathrm{PaO}_{2} / \mathrm{FiO}_{2}$ increased from $173.9 \pm 84.3$ to $273.8 \pm 89.4(P=0.017)$; no statistical differences were observed in the control group. $\mathrm{PaO}_{2}$ and positive end expiratory pressure did not change significantly during the study in both groups.

Figure 1 represents changes in OI between the start and the end of the study of all newborns with pneumonia and of those controls who had at least two TA samples.

SP-B, SP-A, DSPC, and PL amounts are reported in Table 2.

At the start of the study, SP-B, expressed as percentage of PL (SP-B (\%PL)), was significantly higher in pneumonia compared with controls $(P=0.029)$. We also observed a tendency to a higher ELF SP-B and a lower ELF DSPC and ELF PL.

SPs concentration changes between the first and the last collected TAs, expressed as \%PL, are depicted in Figure 2.

In patients with pneumonia SP-B, expressed as $\%$ of PL, was significantly higher at the start of the study compared to the percentage found before extubation (Panel a, $0.99(0.20-1.33) \%$ and $0.05(0.02-0.10) \%$ respectively, $P=0.003)$. Similarly, SP-A $(\% \mathrm{PL})$ ratio was higher at study start than before extubation (Panel c, $0.59(0.39-1.23) \%$ and $0.26(0.13-0.50) \%$ respectively, $P=0.050)$.

In control newborns, from whom SPs could be measured at least two times $(n=7)$, SP-B (\%PL) did not differ during the study period, (Panel b, $0.23(0.03-0.31) \%$ and 0.06

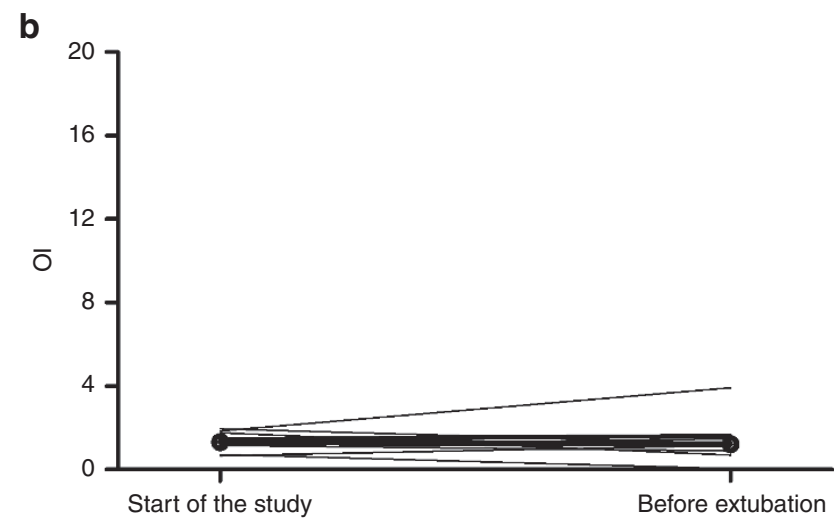

Figure 1. Changes in oxygenation index $(\mathrm{OI})$ at the start of the study and close to the extubation in the two study groups. Bold lines represented the intersection of the two median Ol values of each group. Panel $\boldsymbol{a}$ refers to pneumonia group, $\boldsymbol{b}$ refers to control group. ${ }^{*} P \leq 0.05$. 
Table 2. ELF DSPC, PL, SP-B, and SP-A measured at the start of the study; SP-B and SP-A measured at the start of the study and before extubation, expressed as \% of PL, in the two study groups

\begin{tabular}{|c|c|c|c|}
\hline & Pneumonia group & Control group & $P$ \\
\hline ELF DSPC start of the study $(\mathrm{mg} / \mathrm{ml})$ & $1.90(0.63-4.10)(n=11)$ & $4.13(1.11-5.76)(n=14)$ & 0.286 \\
\hline ELF PL start of the study ${ }^{a}(\mathrm{mg} / \mathrm{ml})$ & $3.21(1.41-6.85)(n=11)$ & $6.82(2.25-10.46)(n=14)$ & 0.193 \\
\hline ELF SP-A start of the study $(\mu \mathrm{g} / \mathrm{ml})$ & $16.99(11.95-44.19)(n=11)$ & $15.99(11.34-41.48)(n=14)$ & 0.815 \\
\hline SP-B (\%PL) start of the study ${ }^{\mathrm{a}}$ & $0.99(0.20-1.33)(n=11)$ & $0.28(0.03-0.58)(n=14)$ & 0.029 \\
\hline SP-B (\%PL) pre-extubation ${ }^{a}$ & $0.05(0.02-0.10)(n=11)$ & $0.06(0.03-0.09)(n=7)$ & 0.821 \\
\hline SP-A (\%PL) pre-extubation ${ }^{a}$ & $0.26(0.13-0.50)(n=11)$ & $0.40(0.14-0.79)(n=7)$ & 0.497 \\
\hline
\end{tabular}

DSPC, disaturated-phosphatidylcholine; ELF, epithelial lining fluid; PL total phospholipids.

${ }^{a}$ Data presented as median (IQR).

a

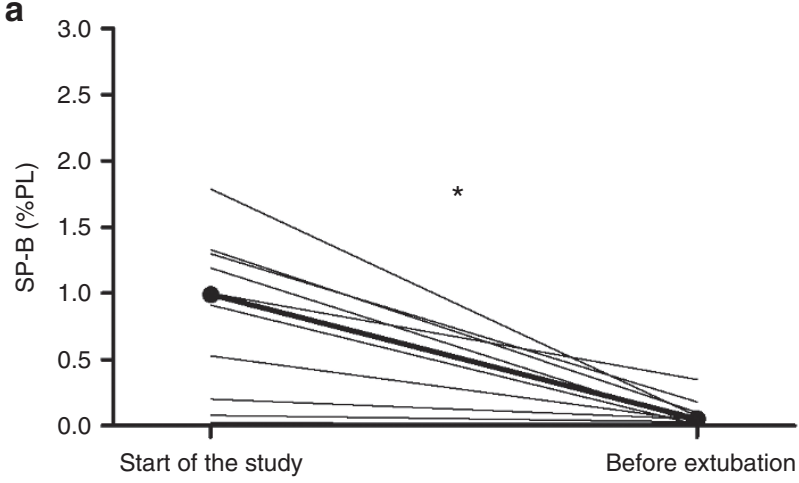

C

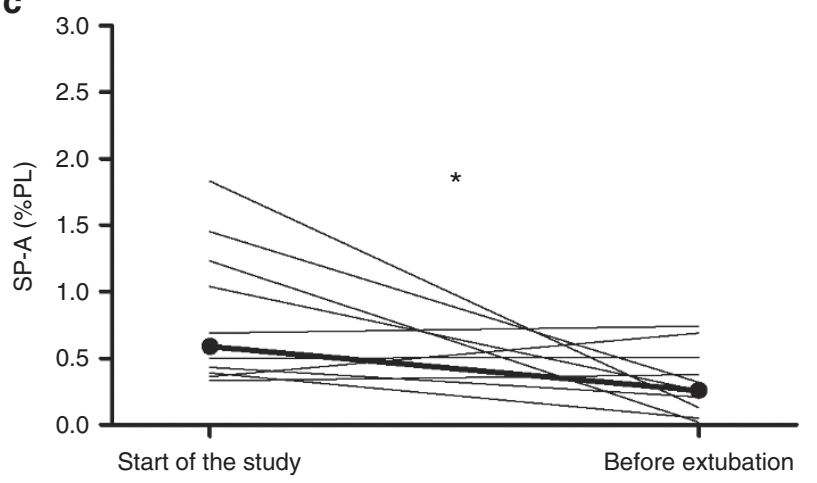

b

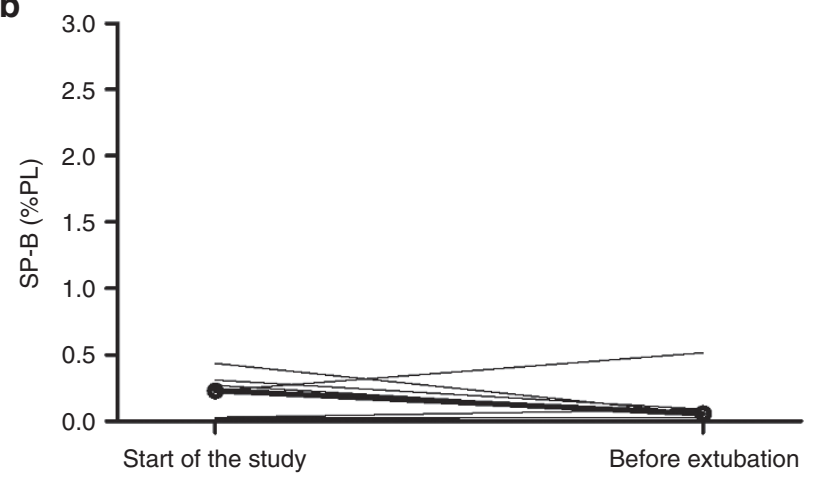

d

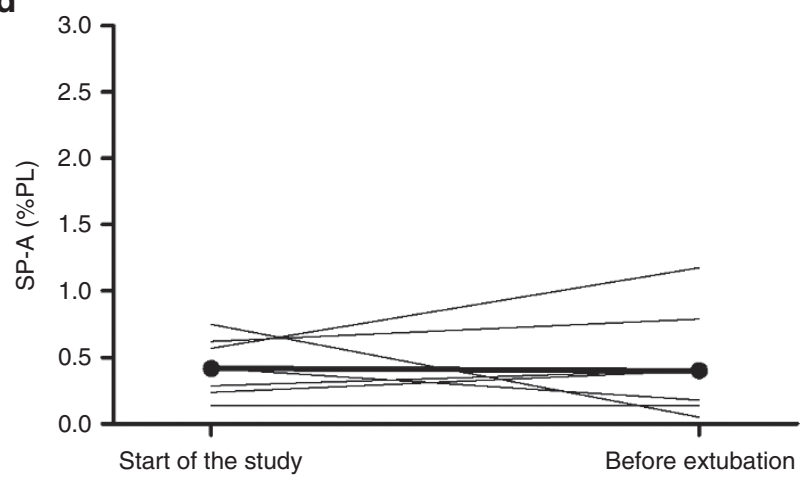

Figure 2. Changes in surfactant protein $(\mathrm{SP})(\% \mathrm{PL})$ ratio in tracheal aspirates collected at the start of the study and close to the extubation in the two study groups. Bold lines represented the intersection of the two median SP (\%PL) ratio values of each group. Panels $\boldsymbol{a}$ and $\boldsymbol{c}$ refer to pneumonia group, $\boldsymbol{b}$ and $\boldsymbol{d}$ to control group. ${ }^{*} P \leq 0.05$.

(0.03-0.09)\%, $P=0.398)$ nor did SP-A (\%PL) (Panel $d, 0.42$ $(0.23-0.62) \%$ and $0.40(0.14-0.79) \%, P=0.866)$ (Figure 2).

DSPC $t_{1 / 2}$ was significantly shorter in the pneumonia group (11.8 (5.5-19.8) h) than in the control group (26.6 (19.3-63.6) h) $(P=0.011)$. Median DSPC pool size (PS) was lower in newborns with pneumonia compared to newborns with no lung disease (10.6 (4.3-30.0) and 36.0 (29.5-84.0) $\mathrm{mg} / \mathrm{kg}$ body weight, $P=0.031)$.

No significant correlations were found between SP amounts at the start of the study and the DSPC $t_{1 / 2}$, PS or OI, both in the pneumonia and control groups.

\section{DISCUSSION}

In this study, SP-B and SP-A amounts in TAs of term newborns with pneumonia were compared with those of newborns with healthy lungs. We also studied the SPs profile during pneumonia clinical course, from the peak of the disease until the clinical improvement and extubation.

Few studies have explored changes in TA SP concentrations during pneumonia in children and no one have presented data corrected for TA dilution (\% of PL and ELF) and/or have related the TA SPs amounts to the severity of respiratory failure $(12,13)$. 


\section{Articles $\mid D^{\prime}$ Aronco et al.}

Expressing SPs levels as \% of PL, we found that in patients with pneumonia, SP-B was significantly higher than in controls at study start and that both SP-B and SP-A decreased, as pulmonary function improved. Expressing data as $\mu \mathrm{g} / \mathrm{ml}$ ELF, we found no significant differences between pneumonia and control patients, although the levels of SP-B tended to be higher and those of DSPC and PL tended to be lower at the start of the study in the pneumonia group. We also found that DSPC was turned over at a faster rate in pneumonia and that DSPC PS was significantly decreased in the pneumonia group.

Based on literature data $(9,16,17)$, we expected to find lower levels of SP-B at the onset of pneumonia and to observe increasing SP-B levels during recovery. Despite the heterogeneity of data, we were instead struck by finding at the onset of pneumonia an excess of SP-B, with respect to PL. It is possible that SP-B excess represents "spent surfactant", that could contribute with factors, like a leak of plasma proteins in the alveolar space (2), or SP-C deficiency (18), to the decreased lung compliance observed at the onset of the pneumonia. The observed SP-B excess could, however, be explained by other mechanisms like increased secretion of SP-B, a slower clearance or a combination of these mechanisms. Finally, it is possible that the excess of SP-B may reflect an accumulation of surfactant subfractions particularly enriched in SP-B (19). The decrease of the SP-B (\%PL) ratio along with clinical and respiratory improvements, were reasonably related with a better oxygenation status, which may represent the result of an improved clearance or a decreased secretion of SP-B.Our data are in line with previous results obtained both in animal studies $(20,21)$ and in newborns after lung injury $(22,23)$. Ikegami et al. (20) found that in healthy mice (with normal lungs) the induction of lung injury by the intratracheal injection of LPS resulted in an increase of DSPC and SP-B, mediated by STAT-3, which is consistent with an acute response to the lung injury. Moreover, our research group observed how, in a murine model of unilateral lung injury, DSPC-palmitate synthesis was faster both in the injured and in the noninjured lungs, compared to the naive control lungs. In this way we proved that, after a local instillation of acid in one bronchus, it is the entire lung system that is involved in responding to the damage and not only a local area of the lung (21). Other mechanisms may have contributed to the elevation of SP-B levels. Increased expression of SPs has been observed both in animals exposed to high levels of inspired oxygen (24) and in mechanically ventilated ones (25), however we hypothesised that a main role could have been played by the infection and be mediated by STAT-3 pathway.

A study performed by Epaud et al. (26) found that elevated levels of mature SP-B peptide in the airspaces of transgenic mice were associated with decreased inflammation following exposure to endotoxins. Based on these findings, a larger study could help us to understand if, in term newborns with pneumonia, higher levels of SP-B are associated with a better clinical outcome and a faster recovery.

The decreased SP-A (\%PL) ratio during resolution of pneumonia could be related either to differences in the turnover of surfactant or to other phenomena related to the nonrespiratory properties of SP-A.

Only two reports have previously described surfactant composition changes during bacterial pneumonia in infants $(12,13)$. Both studies corrected the TA dilution by TA total protein amount; this method is less reliable than the ELF method because of the risk to underestimate the SPs amounts due to the increased alveolar capillary leak during the disease's course (27). Kerr et al. (11) measured SP levels in infants ventilated for respiratory failure resulting from severe respiratory syncytial virus bronchiolitis but TA dilution was, also in this case, corrected by TA total protein amount.

In our study, we used urea to correct for surfactant dilution (28) in the first TA sample, and we expressed SPs concentrations as percentage by weight of total PL in absence of available plasmatic urea level in the second TA. At the end of the study, after the removal of the arterial line, we could not express SPs as ELF, because ethical concerns precluded invasive and unnecessary procedures, such as the collection of plasma to correct for surfactant dilution. We therefore chose to express SPs values as \% of PL because the amount of total PL recovered from TAs did not significantly differ from the start to the end of the study both in the pneumonia and in the control groups (pneumonia: $0.14(0.07-0.25)$ and $0.17(0.09-0.97) \mathrm{mg} / \mathrm{ml}$, $P=0.110$; controls: $0.50(0.20-0.75)$ and $0.39(0.06-0.56) \mathrm{mg} /$ $\mathrm{ml}, P=0.237)$. This implied that they did not affect the SPs $(\% \mathrm{PL})$ ratio and they did not introduce a bias in the comparison within the study groups. A previous study performed in adults with pneumonia, ARDS, or cardiogenic edema showed that in adults with pneumonia the BALF PL and phosphatidylcholine (PC) content were not significantly different compared with controls (2). It is conceivable that the TAs unsaturatedPC derived from inflammatory cells may have contributed to mantain the same amount of TAs PL content in our newborns with pneumonia together with the increase of other surfactant PL classes, either than the sole DSPC (13). Regarding SP-A (\%PL) ratio, Dargaville et al. (10) expressed SP-A content as weight/volume of ELF, and as opposed to our study, they found that ELF SP-A in infants with respiratory syncytial virus bronchiolitis was, at the peak of the disease, significantly lower compared to controls. This conflicting finding can be explained by the different etiology of the disease or by the fact that they measured ELF SP-A in infants with gestational age ranging between $24-41 \mathrm{wk}$.

The administration of a tracer dose of (U- $\left.{ }^{13} \mathrm{C}-\mathrm{PA}\right)$ dipalmitoyl-phosphatidylcholine allowed us to measure the DSPC kinetics, confirming previous findings of our research group (15). We found that in newborns with pneumonia DSPC PS and $t_{1 / 2}$ are, respectively, lower and shorter compared to controls. The faster turnover of TA DSPC in these patients likely reflects the hyperventilation associated with pneumonia and/ or the increased DSPC synthesis. The increased catabolism of DSPC observed in children with pneumonia supports the hypothesis that increasing SP-B levels could represent a compensatory mechanisms of the lung to a damage, as we earlier described in a murine model of unilateral lung injury (21). 
Our study has four major limitations: first, subject number; second, term newborns who served as controls were on mechanical ventilation for a median value of $24 \mathrm{~h}$, which may have alterated the surfactant homeostasis, hiding possible significant differences between healthy and ill newborns; third, ethical limitations prevented the plasma collection close to the extubation and the calculation of SPs in ELF. Finally, to measure DSPC kinetics, we used $\left(\mathrm{U}-{ }^{13} \mathrm{C}-\mathrm{PA}\right)$ dipalmitoylphosphatidylcholine as tracer, in agreement with previous animal studies (29) but without the possibility to prove the actual mixing with the endogenous surfactant pool.

In conclusion, in term newborns with pneumonia SP-B increased with respect to PL and DSPC turned over at a faster rate. Resolution of the disease was associated with restoration of the normal ratio between SP-B and PL.

\section{METHODS}

\section{Study Population}

From January 2011 to December 2013, we prospectively recruited 13 term newborns (37-41 wk GA), up to $10 \mathrm{~d}$ of life with neonatal pneumonia. We also recruited 15 newborns with no lung disease who required mechanical ventilation for elective surgery or neurological impairment. All newborns were admitted to the Neonatal Intensive Care Units of the University of Padua or of the Polytechnic University of Marche, Ancona, Italy.

The diagnosis of pneumonia was based on the 2008 CDC/NHSN (Centers for Disease Control and Prevention/National Healthcare Safety Network) criteria for pneumonia in infants $\leq 1$ y of age (30). These criteria are basically limited to clinically defined pneumonia and are: (i) Chest $\mathrm{x}$-ray showing new or progressive or persistent infiltrate, consolidation or cavitation or pneumatoceles; (ii) Worsening gas exchange (desaturation or rise in oxygen requirement or rise in ventilation demand); (iii) At least three of the following: temperature instability with no other recognized cause; leucopenia $(<4,000 \mathrm{WBC} /$ $\left.\mathrm{mm}^{3}\right)$, or leucocytosis $\left(\geq 15,000 \mathrm{WBC} / \mathrm{mm}^{3}\right)$ with left shift $(\geq 10 \%$ band cells); new onset of purulent sputum, or change in character of sputum, or increased respiratory secretions, or increased suctioning requirements; apnea, tachypnea, nasal flaring with retraction of chest wall or grunting; wheezing, rales or rhonchi; cough; bradycardia $(<100$ beats/min) or tachycardia ( $>170$ beats/min). Diagnosis of bacterial pneumonia was based on these indirect clinical criteria and on the response given by every infant to the antibiotic therapy.

Inclusion criteria were: respiratory failure (newborns with pneumonia) requiring mechanical ventilation with $\mathrm{FiO}_{2}>0.35$, a mean airway pressure $>7 \mathrm{~cm} \mathrm{H}_{2} \mathrm{O}$, and a prediction to be mechanically ventilated for at least $48 \mathrm{~h}$.

Control newborns had no lung disease (no clinical and laboratory signs of infection, normal chest $\mathrm{x}$-ray, $\mathrm{FiO}_{2}<0.30$ ) but required mechanical ventilation as a result of major surgery or neurological impairment leading to poor airway control.

Another inclusion criterion for both groups was the presence, at the start of the study, of an arterial line, placed for invasive arterial blood pressure monitoring and/or arterial blood gas analysis.

Exclusion criteria were: (i) severe congenital malformations; (ii) chromosomal abnormalities; (iii) exogenous surfactant administration in the last $48 \mathrm{~h}$ before the start of the study.

The study protocol was approved by the Local Ethics Committee of the University of Padua and by the Ethics Committee of the Polytechnic University of Marche, and written informed consent was obtained from both parents.

\section{Study Design}

We collected TAs every $6 \mathrm{~h}$ for the first $72 \mathrm{~h}$ and then every $12 \mathrm{~h}$ until extubation in newborn with pneumonia and in controls; $0.5 \mathrm{ml}$ of blood was drawn at the start of the study in EDTA-containing tubes to perform arterial gas analysis and plasma urea determination. If additional parental consent was obtained, we also measured DSPC turnover with a tracer dose of (U- $\left.{ }^{13} \mathrm{C}-\mathrm{PA}\right)$ dipalmitoyl-phosphatidylcholine mixed with $2-5 \mathrm{mg} / \mathrm{kg}$ of Curosurf, used as a spreading agent. Administration of the tracer dose and TA collection and processing were performed as previously reported $(15,31)$. Processed samples were stored at $-80^{\circ} \mathrm{C}$ until analysis. We used TAs instead of BALF because of ethical concerns, since TA suctioning is a routine procedure to clean endotracheal tube whereas BALF are seldomly performed in neonatal intensive care. Moreover, a recent study suggested that TAs surfactant PL composition is similar to that obtained from BALF (32).

Vital and ventilator parameters were recorded every hour. Arterial blood gas analysis were recorded at the start of the study and then at least every $12 \mathrm{~h}$, according to the policy of the two units or when requested by the attending physician. Respiratory failure was assessed by $\mathrm{PaO}_{2} / \mathrm{FiO}_{2}$, OI, and $\mathrm{AaDO}_{2}$, calculated as $\left[\left(\mathrm{MAP} \times \mathrm{FiO}_{20}\right) / \mathrm{PaO}_{2}\right]$ $\times 100$ and $\left[\mathrm{FiO}_{2} \times(760-47)\right]-\left[\left(\mathrm{PaCO}_{2} / 0.8\right)\right]-\mathrm{PaO}_{2}$, respectively.

\section{Analytical Methods}

SP-B and SP-A amounts in TAs were measured by enzyme-linked immunosorbent assay (ELISA) using antihuman SP-B and SP-A antibodies raised in rabbits, produced in our laboratory, and using SP-B and SP-A proteins isolated from BALF of patients with alveolar proteinosis as standards $(19,33)$.

SP-B was measured according to a previously described ELISA technique (34). SP-A content in TAs was determined by a competitive ELISA (19).

DSPC was extracted from TAs according to the method of Bligh and Dyer (35) after the addition of $50 \mu \mathrm{g}$ of internal standard (pentadecanoylphosphatidylcholine, Sigma-Aldrich Italy, Milan, Italy). The lipid extract was reacted with osmium tetroxide and DSPC was then isolated by thin layer chromatography. DSPC fatty acids were hydrolyzed and derivatized as methyl-esters, and DSPC amount was quantified by gas-chromatography (36).

PL in TAs was quantified by measurement of PL phosphorus (37) after their liquid-liquid extraction (35).

Plasma and TA urea was determined with a colorimetric assay (QuantiChrom Urea Assay kit, BioAssay Systems, Hayward, CA) to normalize SP-B, SP-A, DSPC and PL for the ELF volume (28).

${ }^{13} \mathrm{C}$-palmitate enrichment was measured by gas-chromatography-mass-spectrometry (GC-MS, 6890N-5973 inert, Agilent Technologies, Milan, Italy). Results were expressed as mole percent excess referring to a calibration curve $(31,38)$.

\section{Calculation and Statistics}

SP amounts in TAs were expressed as \% of PL or as concentrations in ELF (28). DSPC $t_{1 / 2}$ and PS were calculated as detailed before $(31,38)$.

Data are presented as mean \pm SD or median (IQR) based on the variable distribution. Comparisons within the groups were assessed by Wilcoxon signed-rank test. Comparisons between the two study groups were assessed by Student t test or Mann-Whitney test, according to the variable distribution. Significance was set at $P \leq$ 0.05 . Pearson's correlation analysis was performed to test the association between SP-B (\%PL) ratio, SP-B ELF, SP-A (\%PL) ratio and SP-A ELF measured at the start of the study, with the OI and DSPC $t_{1 / 2}$.

Statistical analysis was performed using PASW Statistics $18.0^{1 / 2}$ for Windows (SPSS, Chicago, IL).

\section{STATEMENT OF FINANCIAL SUPPORT}

No financial support was receveid for this study.

Disclosure: The authors confirm that there is no potential, perceived, or real conflict of interest.

\section{REFERENCES}

1. Gregory TJ, Longmore WJ, Moxley MA, et al. Surfactant chemical composition and biophysical activity in acute respiratory distress syndrome. J Clin Invest 1991;88:1976-81.

2. Günther A, Siebert C, Schmidt R, et al. Surfactant alterations in severe pneumonia, acute respiratory distress syndrome, and cardiogenic lung edema. Am J Respir Crit Care Med 1996;153:176-84. 
3. Kuroki Y, Takahashi M, Nishitani C. Pulmonary collectins in innate immunity of the lung. Cell Microbiol 2007;9:1871-9.

4. Pérez-Gil J. Structure of pulmonary surfactant membranes and films: the role of proteins and lipid-protein interactions. Biochim Biophys Acta 2008;1778:1676-95.

5. Hawgood S, Derrick M, Poulain F. Structure and properties of surfactant protein B. Biochim Biophys Acta 1998;1408:150-60.

6. Clark JC, Wert SE, Bachurski CJ, et al. Targeted disruption of the surfactant protein $\mathrm{B}$ gene disrupts surfactant homeostasis, causing respiratory failure in newborn mice. Proc Natl Acad Sci USA 1995;92:7794-8.

7. Haagsman HP, Diemel RV. Surfactant-associated proteins: functions and structural variation. Comp Biochem Physiol A Mol Integr Physiol 2001;129:91-108.

8. LeVine AM, Whitsett JA. Pulmonary collectins and innate host defense of the lung. Microbes Infect 2001;3:161-6.

9. Avery ME, Mead J. Surface properties in relation to atelectasis and hyaline membrane disease. AMA J Dis Child 1959;97(5, Part 1):517-23.

10. Dargaville PA, South M, McDougall PN. Surfactant abnormalities in infants with severe viral bronchiolitis. Arch Dis Child 1996;75: $133-6$.

11. Kerr MH, Paton JY. Surfactant protein levels in severe respiratory syncytial virus infection. Am J Respir Crit Care Med 1999;159(4 Pt 1):1115-8.

12. LeVine AM, Lotze A, Stanley S, et al. Surfactant content in children with inflammatory lung disease. Crit Care Med 1996;24:1062-7.

13. Todd DA, Marsh MJ, George A, et al. Surfactant phospholipids, surfactant proteins, and inflammatory markers during acute lung injury in children. Pediatr Crit Care Med 2010;11:82-91.

14. Cogo PE, Toffolo GM, Ori C, et al. Surfactant disaturated-phosphatidylcholine kinetics in acute respiratory distress syndrome by stable isotopes and a two compartment model. Respir Res 2007;8:13.

15. Facco M, Nespeca $M$, Simonato $M$, et al. In vivo effect of pneumonia on surfactant disaturated-phosphatidylcholine kinetics in newborn infants. PLoS One 2014;9:e93612.

16. Nogee LM. Genetics of the hydrophobic surfactant proteins. Biochim Biophys Acta 1998;1408:323-33.

17. Nogee LM, de Mello DE, Dehner LP, Colten HR. Brief report: deficiency of pulmonary surfactant protein B in congenital alveolar proteinosis. N Engl J Med 1993;328:406-10.

18. Mulugeta S, Beers MF. Surfactant protein C: its unique properties and emerging immunomodulatory role in the lung. Microbes Infect 2006;8:2317-23.

19. Baritussio A, Alberti A, Quaglino D, et al. SP-A, SP-B, and SP-C in surfactant subtypes around birth: reexamination of alveolar life cycle of surfactant. Am J Physiol 1994;266(4 Pt 1):L436-47.

20. Ikegami M, Falcone A, Whitsett JA. STAT-3 regulates surfactant phospholipid homeostasis in normal lung and during endotoxin-mediated lung injury. J Appl Physiol (1985) 2008;104:1753-60.

21. Lamonica G, Amigoni M, Vedovelli L, et al. Pulmonary surfactant synthesis after unilateral lung injury in mice. J Appl Physiol (1985) 2014;116: $210-5$.
22. Friedrich B, Schmidt R, Reiss I, et al. Changes in biochemical and biophysical surfactant properties with cardiopulmonary bypass in children. Crit Care Med 2003;31:284-90.

23. Griese M, Wilnhammer C, Jansen S, Rinker C. Cardiopulmonary bypass reduces pulmonary surfactant activity in infants. J Thorac Cardiovasc Surg 1999;118:237-44.

24. Nogee LM, Wispé JR, Clark JC, Weaver TE, Whitsett JA. Increased expression of pulmonary surfactant proteins in oxygen-exposed rats. Am J Respir Cell Mol Biol 1991;4:102-7.

25. Woods E, Ohashi T, Polk D, Ikegami M, Ueda T, Jobe AH. Surfactant treatment and ventilation effects on surfactant SP-A, SP-B, and SP-C mRNA levels in preterm lamb lungs. Am J Physiol 1995;269(2 Pt 1):L209-14.

26. Epaud R, Ikegami M, Whitsett JA, Jobe AH, Weaver TE, Akinbi HT. Surfactant protein B inhibits endotoxin-induced lung inflammation. Am J Respir Cell Mol Biol 2003;28:373-8.

27. de Blic J, Midulla F, Barbato A, et al. Bronchoalveolar lavage in children. ERS Task Force on bronchoalveolar lavage in children. European Respiratory Society. Eur Respir J 2000;15:217-31.

28. Dargaville PA, South M, Vervaart P, McDougall PN. Validity of markers of dilution in small volume lung lavage. Am J Respir Crit Care Med 1999;160:778-84.

29. Alberti A, Pettenazzo A, Enzi GB, et al. Uptake and degradation of Curosurf after tracheal administration to newborn and adult rabbits. Eur Respir J 1998;12:294-300.

30. Horan TC, Andrus M, Dudeck MA. CDC/NHSN surveillance definition of health care-associated infection and criteria for specific types of infections in the acute care setting. Am J Infect Control 2008;36:309-32.

31. Torresin M, Zimmermann LJ, Cogo PE, et al. Exogenous surfactant kinetics in infant respiratory distress syndrome: A novel method with stable isotopes. Am J Respir Crit Care Med 2000;161:1584-9.

32. Dushianthan A, Goss V, Cusack R, Grocott MP, Postle AD. Phospholipid composition and kinetics in different endobronchial fractions from healthy volunteers. BMC Pulm Med 2014;14:10.

33. Alberti A, Luisetti M, Braschi A, et al. Bronchoalveolar lavage fluid composition in alveolar proteinosis. Early changes after therapeutic lavage. Am J Respir Crit Care Med 1996;154(3 Pt 1):817-20.

34. Krämer HJ, Schmidt R, Günther A, Becker G, Suzuki Y, Seeger W. ELISA technique for quantification of surfactant protein B (SP-B) in bronchoalveolar lavage fluid. Am J Respir Crit Care Med 1995;152(5 Pt 1):1540-4.

35. Bligh EG, Dyer WJ. A rapid method of total lipid extraction and purification. Can J Biochem Physiol 1959;37:911-7.

36. Cogo PE, Giordano G, Badon T, et al. Simultaneous measurement of the rates of appearance of palmitic and linoleic acid in critically ill infants. Pediatr Res 1997;41:178-82.

37. Bartlett GR. Phosphorus assay in column chromatography. J Biol Chem 1959;234:466-8.

38. Cogo PE, Simonato M, Mariatoffolo G, et al. Dexamethasone therapy in preterm infants developing bronchopulmonary dysplasia: effect on pulmonary surfactant disaturated-phosphatidylcholine kinetics. Pediatr Res 2008;63:433-7. 